\title{
Discrete Time Model for Bond Detection in Thin Layer Materials
}

\author{
M.G.S. Ali ${ }^{a, *}$, N.Z. ElSAYED ${ }^{a}$ AND G.S. HASSAN ${ }^{b}$ \\ ${ }^{a}$ Physics Department, Faculty of Science, Minia University, Minia, Egypt \\ ${ }^{b}$ Physics Department, Assiut University, 71516 Assiut, Egypt
}

(Received January 29, 2010; revised version January 29, 2012; in final form February 22, 2012)

\begin{abstract}
Recursive equations were developed and introduced that enable to calculate the transmitted waves through a multiplicity of layers. The calculations are carried out using $z$-transform technique. The layers are considered lossless and have parallel interface, which are at normal incidence to the field. The multilayered structures are constructed of three aluminum plates connected together with epoxy as the adhesive layers. The method has been successfully used to detect small changes in the thickness of adhesive layers.
\end{abstract}

PACS: $43.60 .+\mathrm{d}$

\section{Introduction}

Several ultrasonic techniques [1-5] have been used to characterize adhesive bonds. The ability to interpret the output signal and relating to joint quality to improve them by computational means, depends much on our understanding the physical phenomena that affect the wave behavior in the material. In this paper, the similarly analogous electrical transmission line equivalent circuits $[6,7]$ can be employed to represent acoustic propagation through thin layers of material, plane-wave propagation being assumed throughout. The $z$-transform technique is applied to the Laplace model to yield a discrete time model of transmitted acoustic wave through thin layers. The model has been used to calculate the force transfer across these layers. The time domain and frequency domain have been computed through very thin adhesive bond. The response for transmission of acoustic waves through adhesive layers and arbitrary input function can be estimated rapidly. The effect of adhesive thickness has been investigated.

\section{Discrete time model}

When using the analogy between electrical and mechanical transmission lines [8] it is known that the transmission matrix of two transmission lines connected in tandem is equal to the product of the individual matrices [9]. This can be generalized to obtain the expression of the transmission matrix for any number of transmission lines connected in tandem. From this, the transfer

* corresponding author; e-mail: mgalal09@yahoo.com of force across an arbitrary number of layers can be obtained [10]. The transfer of force from one material of acoustic impedance $Z_{\mathrm{L}}$ to another of acoustic impedance $Z_{\mathrm{R}}$ can be described in simple terms (analogous to voltage). The received force, $F_{0}$ is given in terms of the incident force, $F_{\mathrm{i}}$, by the expression

$$
\begin{aligned}
& F_{0}=\frac{Z_{\mathrm{R}} F_{\mathrm{i}}}{Z_{\mathrm{L}}+Z_{\mathrm{R}}} \\
& F_{0}=\frac{F_{\mathrm{i}}}{2}\left(1+r_{0}\right),
\end{aligned}
$$

where $r_{0}=\left(Z_{\mathrm{R}}-Z_{\mathrm{L}}\right) /\left(Z_{\mathrm{R}}+Z_{\mathrm{L}}\right)$ is the reflection coefficient in terms of force at boundary between the two media.

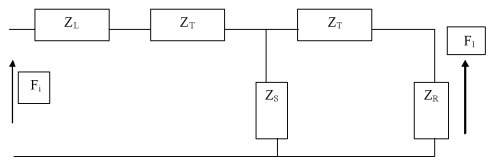

Fig. 1. The equivalent circuit of a mechanical layer.

The transfer of force from a material of impedance $Z_{\mathrm{L}}$ through a layer of impedance $Z_{1}$ to a material of impedance $Z_{\mathrm{R}}$ can be represented in Fig. 1 . In this figure $Z_{\mathrm{S}}$ and $Z_{\mathrm{T}}$ represent the distributed components of the equivalent circuit of a transmission line, however in this case $Z_{\mathrm{S}}$ and $Z_{\mathrm{T}}$ have a mechanical interpretation,

$$
Z_{\mathrm{S}}=\frac{Z_{1}}{\sinh \left(\frac{S X_{1}}{2 V_{1}}\right)}
$$

and 


$$
Z_{\mathrm{T}}=Z_{1} \tanh \left(\frac{S X_{1}}{2 V_{1}}\right),
$$

where $X_{1}$ is the thickness of the acoustic layer, $V_{1}$ is the velocity of the compression wave in the layer material of acoustic impedance $Z_{1}$ and the Laplace variable $S$ is the complex frequency. The term $X_{1} / V_{1}=T_{\mathrm{p}}$ represent the time delay for an acoustic wave to propagate from one face of the acoustic layer of impedance $Z_{1}$ to the other.

For digital computers it is convenient to consider continuous time as being sampled at regular intervals of $T$ seconds, where $T$ is the sampling period. It is clear that a number of discrete time intervals could approximately represent the time delay $m T$ where $m$ is an integer. In terms of the Laplace transform [11] the time delay $\mathrm{e}^{-S X_{1} / V_{1}}$ approximates to $\mathrm{e}^{-S m_{1} T}=z^{-m_{1}}$, where the substitution $z=\mathrm{e}^{S T}$ has been made and the approximate time delay is given in terms of the $z$-transform [12]. By using standard circuit analysis techniques and with the appropriate $z$-transform expression substituted for $Z_{\mathrm{S}}$ and $Z_{\mathrm{T}}$, the force transfer across one layer can be described as

$$
F_{1}=\frac{2 Z_{1} Z_{\mathrm{R}} Z^{-m_{1}} F_{\mathrm{i}}}{\left(Z_{1}+Z_{\mathrm{L}}\right)\left(Z_{1}+Z_{\mathrm{R}}\right)+\left(Z_{1}-Z_{\mathrm{L}}\right)\left(Z_{\mathrm{R}}-Z_{1}\right) Z^{-2 m_{1}}} .
$$

Equation (5) can be interpreted as a recurrence relationship between the sampled versions of $F_{1}(t)$ and $F_{\mathrm{i}}(t)$. The relationship is

$$
\begin{aligned}
& F_{1}(n)=0.5\left[\left(1+r_{0}\right)\left(1+r_{1}\right) F_{\mathrm{i}}\left(n-m_{1}\right)\right] \\
& \quad-2 r_{0} r_{1} F_{1}\left(n-2 m_{1}\right),
\end{aligned}
$$

where $r_{1}=\left(Z_{2}-Z_{1}\right) /\left(Z_{2}+Z_{1}\right)$. The expression for two layers can be interpreted as a recurrence relationship which represents a digital filter relating the $n$-th sample of the transmitted force to the $n$-th and previous samples of the input force wave form and previously calculated force output values

$$
\begin{aligned}
& F_{2}(n)=0.5\left[\left(1+r_{0}\right)\left(1+r_{1}\right)\left(1+r_{2}\right)\right. \\
& \left.\quad \times F_{\mathrm{i}}\left(n-\left(m_{1}+m_{2}\right)\right)\right] \\
& \quad-r_{1} r_{2} F_{2}\left(n-2 m_{2}\right)-r_{0} r_{1} F_{2}\left(n-2 m_{1}\right) \\
& \quad-r_{0} r_{2} F_{2}\left(n-2\left(m_{1}+m_{2}\right)\right) .
\end{aligned}
$$

The digital filters representing the transfer of force across three and four layers can be obtained in the manner. The transfer force across five layers can be written as

$$
\begin{aligned}
& F_{5}(n)=0.5\left[\left(1+r_{0}\right)\left(1+r_{1}\right)\left(1+r_{3}\right)\left(1+r_{4}\right)\left(1+r_{5}\right)\right. \\
& \left.\quad \times F_{\mathrm{i}}\left(n-\left(m_{1}+m_{2}+m_{3}+m_{4}+m_{5}\right)\right)\right] \\
& \quad-A F_{5}\left(n-2 m_{1}\right)-B F_{5}\left(n-2 m_{2}\right) \\
& \quad-C F_{5}\left(n-2 m_{3}\right)-D F_{5}\left(n-2 m_{4}\right) \\
& \quad-E F_{5}\left(n-2 m_{5}\right),
\end{aligned}
$$

where $r_{1}, r_{2}, r_{3}, r_{4}, r_{5}$ are the reflection coefficients

$$
\begin{aligned}
A & =\left(r_{4}+r_{5}\right)\left(r_{0} r_{1} r_{3}+r_{0} r_{2} r_{3}+r_{0}+1\right) \\
& +r_{0}+r_{0} r_{4} r_{5}\left(r_{1}+r_{2}+r_{3}\right)+\left(r_{0} r_{1} r_{2}\right)\left(r_{3}+r_{4} r_{5}\right),
\end{aligned}
$$

$$
\begin{aligned}
B & =\left(r_{0}+r_{1}\right)\left(r_{2}+r_{3}+r_{4}+r_{5}\right) \\
& +\left(r_{4}+r_{5}\right)\left(r_{3}+r_{0} r_{2} r_{3}\right) \\
& +r_{4} r_{5}\left(r_{1} r_{2}+r_{1} r_{3}+r_{0} r_{2}+r_{0} r_{3},\right) \\
C & =\left(r_{0}+r_{1}+r_{2}\right)\left(r_{3}+r_{4}+r_{5}\right) \\
& +r_{2} r_{3}\left(r_{0} r_{1}+r_{4} r_{5}\right) \\
& +\left(r_{4}+r_{5}\right)\left(r_{0} r_{1}+r_{0} r_{1} r_{2}+r_{0} r_{3}+r_{1} r_{3}\right), \\
D & =\left(r_{1}+r_{2}+2 r_{3}\right)\left(r_{4}+r_{5}\right) r_{0} \\
& \times\left(1+r_{1}+r_{1} r_{3}+r_{2} r_{3}\right), \\
E & =\left(r_{2} r_{4} r_{5}+r_{5}\right)\left(r_{0}+r_{0} r_{1}+r_{1}\right) \\
& +\left(r_{2} r_{3} r_{5}+r_{3} r_{4} r_{5}\right)\left(r_{1}+r_{0}\right)+r_{0} r_{1} r_{5}\left(r_{3}+r_{4}\right) \\
& \times\left(\left(r_{2}+2 r_{3}+r_{4}\right) / r_{0} r_{1}\right) .
\end{aligned}
$$

\begin{tabular}{|c|c|c|c|c|}
\hline $\begin{array}{c}\text { Layers } \\
\text { No. }\end{array}$ & Materials & $\begin{array}{l}\text { Density } \\
{\left[\mathrm{kg} / \mathrm{m}^{3}\right]}\end{array}$ & $\begin{array}{c}\text { Velocity } \\
{[\mathrm{m} / \mathrm{s}]}\end{array}$ & $\begin{array}{c}\text { Thickness } \\
\text { [mm] }\end{array}$ \\
\hline layer 1 & $\begin{array}{l}\text { aluminum } \\
{[2024]}\end{array}$ & 2770.0 & 6140 & 1.0 \\
\hline layer 2 & epoxy & 1160 & 2400 & $0.2,0.15$ \\
\hline layer 3 & $\begin{array}{l}\text { aluminum } \\
\text { [7075] }\end{array}$ & 2800 & 6020 & 1.5 \\
\hline layer 4 & epoxy & 1160 & 2400 & $0.2,0.15$ \\
\hline layer 5 & $\begin{array}{l}\text { aluminum } \\
\text { [2024] }\end{array}$ & 2770 & 6140 & 1.0 \\
\hline
\end{tabular}

It is clear that $s$ recursive Eq. (8) can be used to obtain the expression for the force transfer across a five number of layer.

\section{Results}

A Kronecker impulse of unit amplitude was put into the filter. Table shows the materials layers data that have been used in this technique. Assuming the sound propagation time is different for each layer according to the velocity of longitudinal waves. The layered acoustic structure is assumed to be bounded on either side by materials of different characteristic impedance and infinite in extent. The layers are surrounded on the front face by perspex material and by air on the back face. The simulation were carried out with an effective sampling time of 2 ns. An adhesive bonded multilayered structure consists of several layers of different materials connected together with thin adhesive layers. The multi-layered structures are constructed of three aluminum plates connected together with epoxy as the adhesive layers.

The materials data.

TABLE

Figure 2 shows the multi-layered structure that have been used in this technique.

Figure 3 shows the impulse time response function for the five layers arrangement respectively as described in Table with adhesive layers thickness equal to $0.2 \mathrm{~mm}$. 


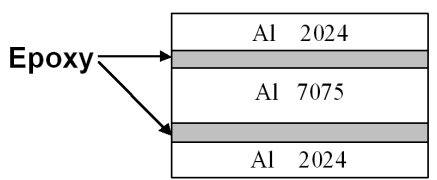

Fig. 2. Multi-layered structure.

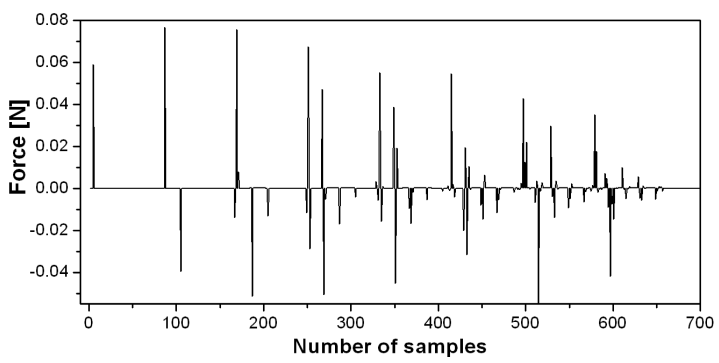

Fig. 3. Time domain impulse response for a pressure wave transmitted through five layers of materials with adhesive thickness layers $0.2 \mathrm{~mm}$.

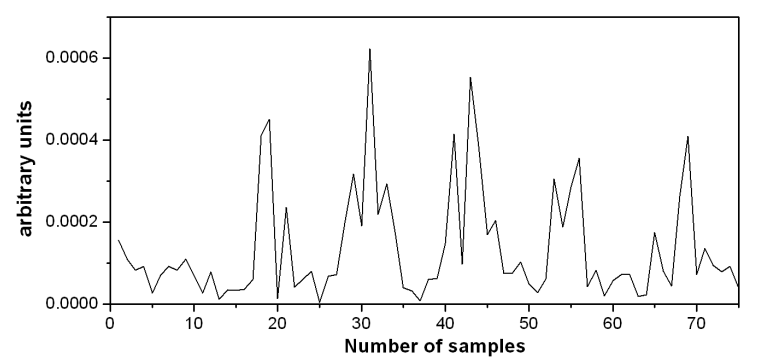

Fig. 4. The frequency response derived from the impulse response of Fig. 3 .

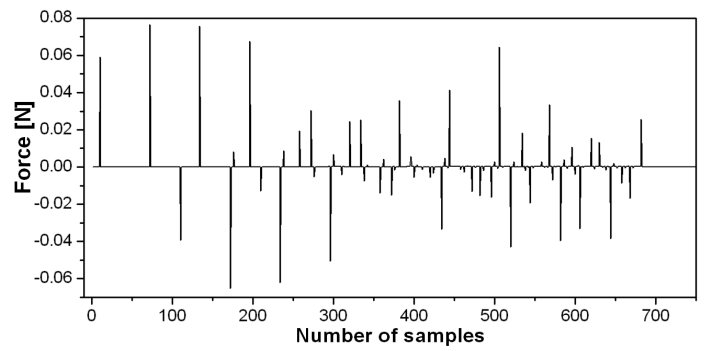

Fig. 5. Transmitted time response for pressures wave through the five layers described in Table with adhesive thickness $0.15 \mathrm{~mm}$.

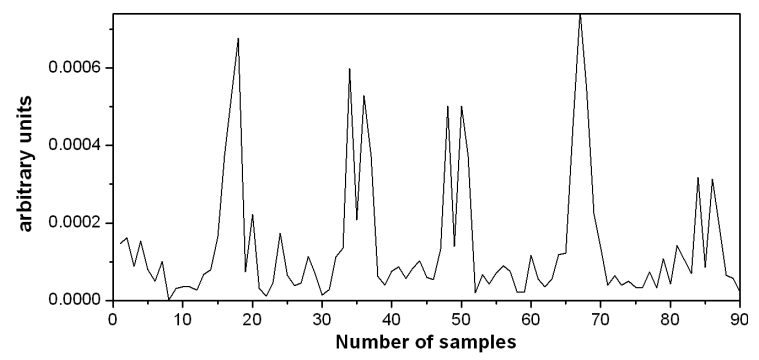

Fig. 6. The frequency domain spectrum of Fig. 5.
The resulting frequency response curve is plotted in Fig. 4. From this figure, it can be seen that the frequency response is periodic with a period equal to the ratio of the velocity of sound propagation to the thickness of the layer.

Figure 5 is a repetiton of the simulation of Fig. 3 but with adhesive thickness 0.15 .

The corresponding frequency response curve is shown in Fig. 6. It is clear from these figures that the shape of the time and frequency domain is sensitive to the thickness of adhesive layers and able to detect small change in the thickness. It is clear from all of these results that calculation using the technique proposed in this paper yields accurate determination of the transmission of pressure waves through several layers of different materials connected together with thin adhesive layers.

\section{Conclusions}

A discrete time model which accurately describes the pressure time response of multilayered acoustic structure has been developed. The $z$-transform has been applied to the Laplace model to yield the impulse response of transmitted force across several layers of different materials connected together with thin adhesive layers. The method has the advantage that the response can be estimated rapidly and detect thin adhesive layers.

\section{References}

[1] R. Adams, P. Cawley, NDT Int. 21, 208 (1988).

[2] W.R Scott, P.F. Gordon, Acoust. Soc. Am. 62, 108 (1977).

[3] L.M. Brekhovskikh, Waves in Layered Media. Applied Mathematics and Mechanics, 2nd ed., Academic, London 1980.

[4] L. Jakevičius, J. Butkus, A. Vladišauskas, Ultragarsas 58, 20 (2006).

[5] E. Maev, I. Severina, S. Bondarenko, G. Chapman, B. O’Neil, F. Severin, R. Maev, Can. J. Appl. Phys. 82, 981 (2004).

[6] M. Redwood, Acoust. Soc. Am. 4, 527 (1961).

[7] O.M. Steutzer, Acoust. Soc. Am. 42, 502 (1967).

[8] G.L. Gooberman, Ultrasonic Theory and Application, Universities Press, London 1968.

[9] F.F. Kuo, Network Analysis and Synthesis, Wiley, New York 1966.

[10] M.G.S. Ali, J. Sound Vibrat. 224, 349 (1999).

[11] A.V. Oppenheim, R.W. Schafer, Digital Signal Processing, Englewood Cliffs, Prentice Hall, NJ 1975.

[12] E. Jury, Theory and Application of the z-Transform Method, Krieger, Florida 1982. 